\title{
ENSAIOS PRELIMINARES EM LABORATÓRIO PARA VERIFICAR A AÇÃO MOLUSCICIDA DE ALGUMAS ESPÉCIES DA FLORA BRASILEIRA*
}

\author{
Nelymar Martineli Mendes * * \\ José Pedro Pereira ** \\ Cecília Pereira de Souza * \\ Maria de Lourdes Lima de Oliveira ** *
}

\begin{abstract}
MENDES, N.M. et al. Ensaios preliminares em laboratório para verificar a ação moluscicida de algumas espécies da flora brasileira. Rev. Saúde públ., S. Paulo, 18:348 - 54, 1984.

RESUMO: Estudou-se em laboratório a atividade moluscicida de 68 extratos de 23 plantas brasileiras. As soluções em água desclorada dos extratos hexânicos e etanólico, nas concentraçōes de 1,10 e $100 \mathrm{ppm}$, foram testadas sobre caramujos adultos e desovas de Biomphalaria glabrata, criados em laboratório. As plantas que demonstraram ação moluscicida na concentração de $100 \mathrm{ppm}$ foram: Arthemisia verlotorum Lamotte, Caesalpinia peltophoroides Benth, Cassia nugosa G.Don., Eclipta alba Hassk, Euphorbia pulcherrima Willd, Euphorbia splendens Bojer, Joannesia princeps Vell, Leonorus sibiricus L., Macrosiphonia guaranitica Muell, Nerium oleander L., Palicourea nicotianaefolia Cham. e Schlech., Panicum maximum M., Rumex crispus L., Ruta graveolens L., e Stryphnodendron barbatiman M.
\end{abstract}

UNITERMOS: Biomphalaria glabrata, condições de laboratório. Moluscicida, extratos vegetais. Esquistossomose mansônica, controle.

\section{INTRODUÇÃO}

A tentativa de controle de criadouros na. turais de Biomphalaria glabrata (Say, 1818), hospedeiro intermediário da Schistosoma mansoni, através de moluscicidas, tem sido uma das maneiras de se combater a esquistossomose. Visando a possibilidade de obten. ção de produtos com alto teor moluscicida, extraídos de vegetais tipicamente nacionais, alguns estudiosos vêm dedicando-se à pesquisa de plantas regionais. Amorim e Pessoa. (1962), Barbosa e Mello ${ }^{2}$ (1969), Sousa e col. ${ }^{10,11}(1970,1974)$, Silva e col. ${ }^{9}(1971)$ e Rouquayrol e col..$^{6,7,8}(1972,1973,1980)$ estudaram plantas do nordeste brasileiro. Pereira e col. ${ }^{4,5}(1974,1978)$ testaram as propriedades moluscicidas do Anacardium occidentale L. (cajueiro) e da Euphorbia cotinifolia L. (roxinha), originários de Fortaleza-CE e de Belo Horizonte-MG, respectivamente. Kloos e McCullough ${ }^{3}$ (1981) fizeram um trabalho de revisão sobre moluscici. das vegetais.

No presente trabalho, foram estudadas em laboratório 23 espécies de plantas brasileiras, objetivando encontrar novas drogas com propriedades moluscicidas, que venham a se tornar produtos de preço mais baixo do que os existentes no mercado, para fazer face às campanhas epidemiológicas de Saúde Pública em nosso país.

\footnotetext{
* Trabalho parcialmente subvencionado pelo Conselho Nacional de Desenvolvimento Científico e Tecnológico (CNPq) - Processo nọ 2222.8.092/80.

**Do Centro de Pesquisas "René Rachou" - FIOCRUZ - Caixa Postal 1743 - 30.000 - Belo Horizonte, MG.

*** Bolsista do CNPq.
} 
MENDES, N. M. et al. Ensaios preliminares em laboratório para verificar a ação moluscicida de algumas espécies da flora brasileira. Rev. Saúde públ., S. Paulo, 18:348 - 54, 1984.

\section{MATERIAL E MÉTODOS}

Os vegetais coletados no campo foram expostos ao ar livre, para secagem, em períodos variando de um a três meses. Depois de triturados, foram submetidos a extrações com hexana e álcool etílico no aparelho de Soxh. let durante $72 \mathrm{~h}, \mathrm{e}$, posteriormente evaporados os solventes. A Tabela 1 mostra espécie, família, nome vulgar e procedência de cada planta.

As "soluções-mãe" foram preparadas dissolvendo-se $50 \mathrm{mg}$ de cada extrato em $0,1 \mathrm{ml}$ de solvente universal (solução de Ritchie) e completando-se o volume para $500 \mathrm{ml}$ com água de torneira desclorada com tiossulfato de sódio a $2 \%$. As soluções a 1 e $10 \mathrm{ppm}$ $(\mathrm{ppm}=\mathrm{mg} / 1)$ foram preparadas através de diluição da "solução-mãe".

Usaram-se caramujos adultos e desovas de B.glabrata, originários da Pampulha - Belo Horizonte, $\mathrm{MGe}$ criados em laboratório. As conchas dos caramujos mediam 10-12 mm de diâmetro e as desovas tinham de 0.1 dia de idade.

Dez caramujos e duas a quatro desovas foram expostos a concentrações de 1,10 e 100 $\mathrm{ppm}$ de cada extrato, durante $24 \mathrm{~h}$. Como controle, dez exemplares e duas a quatro desovas permaneceram somente em água desclorada. A temperatura das soluções foram medidas no início e final de cada experiên. cia e variou de 25 a $28^{\circ} \mathrm{C}$.

Diariamente, durante quatro dias após a exposição, trocava-se a água dos frascos contendo os moluscos e desovas, retirando-se e anotando-se o número de caramujos e embriōes mortos. Para os moluscos sobreviven. tes era colocada alface fresca. As desovas foram observadas em microscópio estereoscópico e os embriōes sobreviventes ficaram em observação até a eclosão.

Baseado na WHO $/ 65^{13}$, que especifica normas para testes com moluscicidas diversos, observou-se, para avaliação da mortalidade nas três concentrações, o critério já usa-

T A B E L A 1

Plantas brasileiras testadas como moluscicidas

\begin{tabular}{lll}
\hline \multicolumn{1}{c}{ Espécie-Familia } & Nome vulgar & Procedência \\
\hline Ampelozizyphus amazonicus Ducke - Rhamnacea & Saracura & Amazonia \\
Arthemisia verlotorum Lamotte - Composta & artemisia & Ouro Preto-MG \\
Caesalpinia peltophoroides Benth-Cesalpinácea & sibipiruna & Belo Horizonte-MG \\
Cassia rugosa G. Don - Cesalpinácea & boi-gordo & Teófilo Otoni-MG \\
Conium maculatum L. - Umbelífera & cicuta & Belo Horizonte-MG \\
Cupania vernalis Camb. - Sapindácea & camboatá & Belo Horizonte-MG \\
Eclipta alba Hassk - Composta & erva de botão & Belo Horizonte-MG \\
Euphorbia pulcherrima Willd - Euphorbiácea & bico de papagaio & Belo Horizonte-MG \\
Euphorbia splendens Bojer - Euphorbiácea & coroa de Cristo & Belo Horizonte-MG \\
Jatropha curcas L. - Euphorbiácea & pinhẫo & Comercinho-MG \\
Joannesia princeps Vell - Euphorbiácea & fruta de arara & Belo Horizonte-MG \\
Leonurus sibiricus L. - Labiada & erva macaé & Ouro Preto-MG \\
Macrosiphonia guaranitica Muell - Apocinácea & babado de N. Sra. & Belo Horizonte-MG \\
Nerium oleander L. - Apocinácea & espirradeira & Belo Horizonte-MG \\
Paepalanthus lamarcku Kunth - Eriocaulácea & capim manso & Ouro Preto-MG \\
Palicourea nicotianaefolia Cham. - Rubiácea & erva de rato & Vespasiano-MG \\
Panicum maximum M. - Leguminosa & capim-guiné & Belo Horizonte-MG \\
Pereskia aculeata Mill - Cactácea & ora-pro-nobis & Ouro Preto-MG \\
Philondendron imbe Schott - Arácea & cipó-imbé & Belo Horizonte-MG \\
Rumex crispus L. - Poligonácea & labaça & Ouro Preto-MG \\
Ruta graveolens L. - Rutácea & arruda & Belo Horizonte-MG \\
Solanum nigrum L. - Solanácea & erva de bicho & Comercinho-MG \\
Stryphnodendron barbatiman M. - Leguminosa & barbatimão & Belo Horizonte-MG \\
\hline & & \\
\hline
\end{tabular}


MENDES, N. M. et al. Ensaios preliminares em laboratório para verificar a ação moluscicida de algumas espécies da flora brasileira. Rev. Saúde públ., S. Paulo, 18:348 - 54, 1984.

do em outras experiências, que considera: a) inativo de 0 a $30 \%$ de mortalidade; b) parcialmente ativo de 40 a $60 \%$ de mortalidade; e c) ativo de 70 a $100 \%$ de mortalidade. Tal critério difere em parte do elaborado pela $\mathrm{WHO} / 83^{14} *$, publicado posteriormente à realização deste trabalho, que estabelece uma linha de pesquisa sobre moluscicida de origem vegetal, na qual, a planta moluscicida só deve ser considerada ativa quando obtiver $90 \%$ de mortalidade nas concentraçőes de 20 ppm para extratos e 100 ppm para o vegetal bruto.

\section{RESULTADOS}

Os resultados dos ensaios biológicos com extratos hexânico e etanólico sobre caramu. jos adultos e desovas de $B$. glabrata nas concentrações de 1,10 e $100 \mathrm{ppm}$ estão suma. rizados nas Tabelas 2 e 3 .

Como pode ser visto na Tabela 2 , dos 34

T A B E L 2

Atividade moluscicida de extratos hexânicos de plantas testadas sobre caramujos adultos e desovas de $B$. glabrata

\begin{tabular}{|c|c|c|c|c|c|c|c|c|}
\hline \multirow{2}{*}{\multicolumn{2}{|c|}{ Espécie }} & \multirow{2}{*}{$\begin{array}{l}\text { Parte } \\
\text { testada }\end{array}$} & \multicolumn{3}{|c|}{ Caramujos adultos } & \multicolumn{3}{|c|}{ Desovas } \\
\hline & & & ppm & 10 ppm & 100 ppm & $1 \mathrm{ppm}$ & 10 ppm & $100 \mathrm{ppm}$ \\
\hline 1. & amazonicus & folhas & $3 / 10$ & $0 / 10$ & $1 / 10$ & $0 / 103$ & $0 / 109$ & $0 / 135$ \\
\hline l. & amazonicus & caule & $/ 10$ & 110 & $2 / 10$ & $0 / 109$ & $0 / 131$ & $0 / 127$ \\
\hline A. & verlotorum & folhas & $0 / 10$ & $0 / 10$ & $0 / 10$ & $0 / 126$ & $0 / 108$ & $72 / 72$ * \\
\hline C. & peltophoroides & folhas & $0 / 10$ & $0 / 10$ & $7 / 10^{*}$ & $0 / 124$ & $0 / 122$ & $112 / 112^{*}$ \\
\hline C. & peltophoroides & casca do caule & $1 / 10$ & $1 / 10$ & $0 / 10$ & $0 / 78$ & $0 / 55$ & 6/91 \\
\hline C. & peltophoroides & raízes & $0 / 10$ & $0 / 10$ & $0 / 10$ & $0 / 130$ & $0 / 140$ & $0 / 121$ \\
\hline C. & rugosa & casca do caule & $0 / 10$ & $1 / 10$ & $7 / 10^{*}$ & $0 / 104$ & $0 / 91$ & $63 / 103 * *$ \\
\hline C. & maculatum & folhas/galhos & $0 / 10$ & $2 / 10$ & $0 / 10$ & $1 / 120$ & $0 / 116$ & $0 / 115$ \\
\hline$C$. & vernalis & folhas & $0 / 10$ & $0 / 10$ & $0 / 10$ & $0 / 146$ & $3 / 133$ & $32 / 152$ \\
\hline$E$. & alba & folhas/galhos & $0 / 10$ & $3 / 10$ & $3 / 10$ & $0 / 122$ & $2 / 111$ & $0 / 144$ \\
\hline$E$. & pulcherrima & folhas & $0 / 10$ & $0 / 10$ & $0 / 10$ & $0 / 153$ & $3 / 118$ & $0 / 166$ \\
\hline$E$. & pulcherrima & flores & $0 / 10$ & $0 / 10$ & $1 / 10$ & $0 / 194$ & $0 / 225$ & $285 / 285^{*}$ \\
\hline$E$. & splendens & caule & $0 / 10$ & $4 / 10 *$ & $10 / 10^{*}$ & $0 / 142$ & $11 / 180$ & $187 / 187^{*}$ \\
\hline$E$. & splendens & casca do caule & $0 / 10$ & $2 / 10$ & $10 / 10^{*}$ & $0 / 130$ & $0 / 123$ & $165 / 165 *$ \\
\hline E. & splendens & folhas & $0 / 10$ & $0 / 10$ & $7 / 10^{*}$ & $0 / 133$ & $0 / 102$ & $0 / 134$ \\
\hline$J$ & curces & folhas/frutos & $0 / 10$ & $0 / 10$ & $0 / 10$ & $0 / 49$ & $0 / 57$ & $0 / 82$ \\
\hline$J$ & princeps & sementes & $1 / 10$ & $0 / 10$ & $2 / 10$ & $0 / 70$ & $0 / 69$ & $0 / 85$ \\
\hline$L$ & sibiricus & raízes & $0 / 10$ & $0 / 10$ & $9 / 10^{*}$ & $0 / 184$ & $0 / 176$ & $172 / 172 *$ \\
\hline$M$. & guaranitica & caule & $0 / 10$ & $0 / 10$ & $5 / 10 * *$ & $0 / 68$ & $0 / 53$ & 3/ 74 \\
\hline$M$. & guaranitica & folhas & $0 / 10$ & $1 / 10$ & $10 / 10^{*}$ & $0 / 131$ & $0 / 164$ & $0 / 188$ \\
\hline$M$. & guaranitica & flores & $0 / 10$ & $0 / 10$ & $10 / 10^{*}$ & $0 / 168$ & $0 / 205$ & $141 / 141 *$ \\
\hline$N$. & oleander & folhas & $0 / 10$ & $0 / 10$ & $0 / 10$ & $0 / 83$ & $0 / 113$ & $0 / 141$ \\
\hline$N$. & oleander & caule & $0 / 10$ & $0 / 10$ & $10 / 10^{*}$ & $0 / 107$ & $0 / 112$ & $0 / 111$ \\
\hline$P$. & lamarcku & flores & $0 / 10$ & $0 / 10$ & $0 / 10$ & $1 / 117$ & $0 / 121$ & $3 / 102$ \\
\hline$P$. & nicotianaefolia & folhas & $1 / 10$ & $1 / 10$ & $0 / 10$ & $0 / 43$ & $0 / 49$ & $0 / 74$ \\
\hline$P$. & maximum & raízes & $0 / 10$ & $1 / 10$ & $1 / 10$ & $0 / 129$ & $13 / 106$ & $3 / 109$ \\
\hline$P$. & maximum & folhas & $0 / 10$ & $1 / 10$ & $8 / 10$ & $0 / 123$ & $0 / 115$ & $1 / 149$ \\
\hline$P$. & aculeata & raízes & $0 / 10$ & $0 / 10$ & $1 / 10$ & $0 / 122$ & $0 / 121$ & $0 / 137$ \\
\hline$P$. & imbe & caule & $0 / 10$ & $0 / 10$ & $0 / 10$ & $0 / 112$ & $0 / 102$ & $0 / 86$ \\
\hline$P$. & imbe & casca do caule & $0 / 10$ & $0 / 10$ & $3 / 10$ & $0 / 176$ & $0 / 128$ & $0 / 141$ \\
\hline$R$. & crispus & raízes & $0 / 10$ & $0 / 10$ & $1 / 10$ & $0 / 75$ & $0 / 116$ & $0 / 111$ \\
\hline$R$. & graveolens & caule/folhas & $6 / 10 * *$ & $4 / 10^{*}$ & $9 / 10^{*}$ & $1 / 106$ & $0 / 92$ & $0 / 93$ \\
\hline$S$ & nigrum & folhas & $0 / 10$ & $0 / 10$ & $0 / 10$ & 0/ 57 & $0 / 60$ & $0 / 47$ \\
\hline$S$ & barbatiman & caule & $0 / 10$ & $0 / 10$ & $10 / 10^{*}$ & $0 / 44$ & $1 / 67$ & $61 / 61 *$ \\
\hline
\end{tabular}

* Ativo para caramujos e/ou desovas

** Parcialmente ativo para caramujos ou desovas

* I Reunião de pesquisadores brasileiros sobre moluscicidas naturais realizada no Centro de Pesquisas "René Rachou", FIOCRUZ, Belo Horizonte-MG, 26-27/01/83. 
MENDES, N. M. et al. Ensaios preliminares em laboratório para verificar a ação moluscicida de algumas espécies da flora brasileira. Rev. Saúde públ., S. Paulo, 18:348 • 54, 1984.

extratos hexânicos testados, $12(35,3 \%)$ mostraram atividade moluscicida para caramujos adultos e $8(23,5 \%)$ para desovas em concentraçס̄es de $100 \mathrm{ppm}$. Um extrato hexânico $(2,9 \%)$ foi parcialmente ativo na con. centração de $1 \mathrm{ppm}$, dois $(5,8 \%)$ nas concentraçס̃es de $10 \mathrm{ppm}, \mathrm{e}, \mathrm{um}(2,9 \%)$ na concentração de $100 \mathrm{ppm}$ para caramujos adultos; apenas um, na concentração de $100 \mathrm{ppm}$, foi parcialmente ativo para desovas. Na Tabela 3 , dos 34 extratos etanólicos, $10(29,4 \%)$ foram ativos para caramujos e $3(8,8 \%)$ para desovas nas concentrações de $100 \mathrm{ppm}$. Um extrato etanólico $(2,9 \%)$ foi parcialmente ativo para caramujos adultos nas concentraçóes de 1 e 10 ppm, $3(8,8 \%)$ na concentra. ção de 100 ppm e um para desovas na concentração de $100 \mathrm{ppm}$.

T A B E L A 3

Atividade moluscicida de extratos etanólicos de plantas testadas sobre caramujos adultos e desovas de B. glabrata

\begin{tabular}{|c|c|c|c|c|c|c|c|}
\hline \multirow{2}{*}{ Espécie } & \multirow{2}{*}{$\begin{array}{l}\text { Parte } \\
\text { testada }\end{array}$} & \multicolumn{3}{|c|}{ Caramujos aduitos } & \multicolumn{3}{|c|}{ Desovas } \\
\hline & & $1 \mathrm{ppm}$ & $10 \mathrm{nnm}$ & 100 ppm & 1 ppm & & $100 \mathrm{ppm}$ \\
\hline $\begin{array}{l}\text { A. amazonicus } \\
\text { A. amazonicus } \\
\text { A. verlotorum } \\
\text { C. peltophoroides } \\
\text { C. peltophoroides } \\
\text { C. peltophoroides } \\
\text { C. rugosa } \\
\text { C. maculatum } \\
\text { C. vernalis } \\
E . \text { alba } \\
E . \text { pulcherrime } \\
E . \text { pulcherrime } \\
E . \text { splendens } \\
E . \text { splendens } \\
E . \text { splendens } \\
J . \text { curcas } \\
J . \text { princeps } \\
\text {. sibiricus } \\
M . \text { guaranitica } \\
M . \text { guaranitica } \\
M . \text { guaranitica } \\
N . \text { oleander } \\
N . \text { oleander } \\
P . \text { lamarcku } \\
P . \text { nicotionaefolia } \\
P . \text { maximum } \\
P . \text { maximum } \\
P . \text { aculeata } \\
P . \text { imbe } \\
P . \text { imbe } \\
R . \text { crispus } \\
R . \text { graveolens } \\
S . \text { nigrum } \\
S . \text { barbatiman }\end{array}$ & $\begin{array}{l}\text { folhas } \\
\text { caule } \\
\text { folhas } \\
\text { folhas } \\
\text { casca do caule } \\
\text { raízes } \\
\text { casca do caule } \\
\text { folhas/galhos } \\
\text { folhas } \\
\text { folhas/galhos } \\
\text { folhas } \\
\text { flores } \\
\text { caule } \\
\text { casca do caule } \\
\text { folhas } \\
\text { folhas/frutos } \\
\text { sementes } \\
\text { raízes } \\
\text { caule } \\
\text { folhas } \\
\text { flores } \\
\text { folhas } \\
\text { caule } \\
\text { flores } \\
\text { folhas } \\
\text { raízes } \\
\text { folhas } \\
\text { raízes } \\
\text { caule } \\
\text { casca do caule } \\
\text { raízes } \\
\text { caule/folhas } \\
\text { folhas } \\
\text { caule }\end{array}$ & $\begin{array}{l}0 / 10 \\
0 / 10 \\
1 / 10 \\
5 / 10 \\
1 / 10 \\
0 / 10 \\
0 / 10 \\
1 / 10 \\
0 / 10 \\
2 / 10 \\
0 / 10 \\
0 / 10 \\
1 / 10 \\
0 / 10 \\
0 / 10 \\
0 / 10 \\
1 / 10 \\
3 / 10 \\
3 / 10 \\
2 / 10 \\
1 / 10 \\
0 / 10 \\
0 / 10 \\
0 / 10 \\
0 / 10 \\
0 / 10 \\
0 / 10 \\
0 / 10 \\
0 / 10 \\
0 / 10 \\
0 / 10 \\
3 / 10 \\
0 / 10 \\
0 / 10\end{array}$ & $\begin{array}{l}0 / 10 \\
0 / 10 \\
0 / 10 \\
6 / 10 \\
2 / 10 \\
2 / 10 \\
0 / 10 \\
1 / 10 \\
0 / 10 \\
0 / 10 \\
0 / 10 \\
0 / 10 \\
0 / 10 \\
0 / 10 \\
0 / 10 \\
1 / 10 \\
2 / 10 \\
0 / 10 \\
3 / 10 \\
2 / 10 \\
0 / 10 \\
0 / 10 \\
0 / 10 \\
0 / 10 \\
0 / 10 \\
0 / 10 \\
0 / 10 \\
0 / 10 \\
0 / 10 \\
0 / 10 \\
0 / 10 \\
0 / 10 \\
0 / 10 \\
0 / 10\end{array}$ & $\begin{array}{c}0 / 10 \\
0 / 10 \\
9 / 10^{*} \\
8 / 10^{*} \\
9 / 10^{*} \\
5 / 10^{* *} \\
2 / 10 \\
0 / 10 \\
0 / 10 \\
8 / 10^{*} \\
0 / 10 \\
0 / 10 \\
9 / 10^{*} \\
10 / 10^{*} \\
10 / 10^{*} \\
0 / 10 \\
10 / 10^{*} \\
10 / 10^{*} \\
2 / 10 \\
4 / 10^{*} \\
1 / 10 \\
0 / 10 \\
1 / 10 \\
0 / 10 \\
6 / 10^{*} \\
0 / 10 \\
0 / 10 \\
0 / 10 \\
0 / 10 \\
0 / 10 \\
0 / 10 \\
10 / 10^{*} \\
0 / 10 \\
0 / 10\end{array}$ & $\begin{array}{c}0 / 80 \\
0 / 92 \\
0 / 111 \\
0 / 95 \\
2 / 93 \\
0 / 43 \\
2 / 77 \\
13 / 87 \\
0 / 102 \\
0 / 109 \\
0 / 153 \\
0 / 145 \\
0 / 97 \\
1 / 92 \\
1 / 97 \\
0 / 115 \\
30 / 78 \\
0 / 113 \\
2 / 74 \\
0 / 212 \\
0 / 212 \\
0 / 149 \\
0 / 114 \\
3 / 179 \\
0 / 99 \\
0 / 142 \\
0 / 123 \\
0 / 171 \\
0 / 94 \\
0 / 145 \\
0 / 52 \\
4 / 109 \\
0 / 130 \\
1 / 67\end{array}$ & $\begin{array}{l}0 / 99 \\
0 / 95 \\
0 / 96 \\
0 / 133 \\
0 / 54 \\
0 / 69 \\
1 / 112 \\
1 / 152 \\
0 / 136 \\
3 / 80 \\
0 / 128 \\
0 / 184 \\
0 / 118 \\
6 / 67 \\
0 / 112 \\
0 / 92 \\
4 / 96 \\
0 / 100 \\
0 / 61 \\
0 / 238 \\
0 / 160 \\
0 / 109 \\
0 / 121 \\
0 / 142 \\
1 / 82 \\
0 / 140 \\
2 / 106 \\
0 / 112 \\
0 / 115 \\
0 / 96 \\
0 / 51 \\
3 / 105 \\
0 / 105 \\
0 / 92\end{array}$ & $\begin{array}{c}0 / 80 \\
0 / 85 \\
0 / 120 \\
0 / 77 \\
0 / 59 \\
2 / 61 \\
96 / 96^{*} \\
2 / 118 \\
0 / 123 \\
0 / 105 \\
4 / 149 \\
0 / 158 \\
9 / 95 \\
0 / 80 \\
0 / 106 \\
0 / 103 \\
114 / 114 * \\
0 / 101 \\
0 / 61 \\
0 / 220 \\
48 / 144 \\
0 / 138 \\
0 / 100 \\
1 / 140 \\
2 / 85 \\
0 / 117 \\
0 / 110 \\
0 / 142 \\
0 / 115 \\
0 / 125 \\
78 / 78^{*} \\
54 / 104^{*} \\
0 / 65 \\
2 / 93\end{array}$ \\
\hline
\end{tabular}

* Ativo para caramujos e/ou desovas

* Parcialmente ativo para caramujos ou desovas 
MENDES, N. M. et al. Ensaios preliminares em laboratório para verificar a ação moluscicida de algumas espécies da flora brasileira. Rev. Saúde públ., S. Paulo, 18:348 · 54, 1984.

\section{DISCUSSÃO}

Amorim e Pessoa ${ }^{1}$ (1962) estudaram 9 espécies de plantas da flora alagoana, obtendo extrações em concentrações de 500,1.000 e $2.000 \mathrm{ppm}$ com a parte do vegetal colocada na água e/ou macerada com água, em almo. fariz. Nas concentrações de $1.000 \mathrm{ppm}$ a Paullinia pinnata L. (cruapé) e a Stenolobium velutinum $\mathrm{B}$ th (tingui) revelaram apreciáveis efeitos moluscicidas, e a Piptadenia macrocarpa Bth (angico-do-campo) e o gravatá-açu mostraram possuir menor ação sobre B.glabrata.

Barbosa e Mello ${ }^{2}$ (1969) testaram material de duas plantas do nordeste brasileiro, triturado no almofariz e seco na estufa a 569 C. As DL 50 obtidas da Magonia pubes. cens (timbó) foram de 6,7 e 7,2 ppm para $B$. glabrata e $B$. straminea, respectivamente.

Pereira e Souza ${ }^{4}$ (1974) obtiveram CL 50 de $1,4 \mathrm{ppm}$ do extrato hexânico da cas. ca da castanha do Anacardium occidentale L. sobre $B$. glabrata .

Rouquayrol e col. ${ }^{8}$ (1980) testaram hidrolatos de 82 espécies de plantas e encontraram 12 com considerável atividade mo. luscicida.

Souza e col. ${ }^{12}$ (1984) apresentaram os dados relativos à atividade moluscicida de 84 plantas brasileiras sobre $B$. glabrata, sen. do que a Mikania hirsutissima DC e a Qualea multiflora Mart. foram letais a caramujos adultos na concentração de $10 \mathrm{ppm}$.

Rouquayrol e col. ${ }^{6}{ }^{7}(1972,1973)$, Sil. va e col. ${ }^{9}(1971)$, Sousa e col. ${ }^{11}$ (1970) e Sousa e Rouquayrol ${ }^{10}$ (1974) usaram extra. tos aquosos e alcoólicos do material fervido e/ou triturado de 122 vegetais do nordeste brasileiro, sobre $B$. glabrata e/ou B. straminea nas concentrações de 2 a 10.000 ppm. Rouquayrol e $\mathrm{col}^{7}$ evidenciaram que a $\mathrm{Pi}$ thecelobium multiflorum Bth. (canafístula) apresentou para caramujos adultos as CL 50 e CL 90 situadas em torno de 3,0 e 4,9 ppm, e, para as desovas, em torno de 13 e $21 \mathrm{ppm}$, respectivamente.

A Euphorbia tenuifolia (erva de cabra) ${ }^{9}$ e a Euphorbia gymnoclada Boiss (avelós) ${ }^{11}$ não apresentaram atividade moluscicida na concentração de $1.000 \mathrm{ppm}$ sobre B. Glabrata e $B$. straminea. A Caesalpinia ferrea Mart. ex. Tull (Jucá) ${ }^{11}$, a Caesalpinia pyramidales Tull (Catingueira) ${ }^{6}$ e a Stryphnodendron coriaceum $\mathrm{Bth}^{11}$ mostraram ativas a 10.000 ppm sobre $B$. glabrata e/ou $B$. straminea.

As concentrações letais (CL 50 e CL90) do extrato hexânico da Euphorbia cotinifolia L. foram 1,2 e 2,4 ppm, respectivamente, sobre B. glabrata ${ }^{5}$ (1978).

No presente trabalho, foram ativas a 100 ppm sobre B. glabrata: Euphorbia pulcherrima Willd, Euphorbia splendens Bojer, Caesalpinia peltophoroides Bth e Stryphnodendron barbatiman $\mathrm{M}$.

Os resultados obtidos para Euphorbia, Caesalpinia e Stryphnodendron, neste experimento, diferenciaram dos obtidos pelos autores acima citados $6,9,11$, em virtude de ter sido usada outra metodologia.

No presente trabalho, como nos anteriores (Pereira e Souza ${ }^{4}, 1974$ - Pereira e col. ${ }^{5}$, 1978), optou-se pelo uso simultâneo dos extratos hexânicos e etanólicos para melhor avaliar em termos de comparação a eficiên. cia dos vegetais estudados. Pelos resultados obtidos, observou-se que $38,2 \%$ dos extratos hexânicos e $38,2 \%$ dos extratos etanólicos, em concentração de 100 ppm, apresentaram-se ativos ou parcialmente ativos sobre caramujos adultos. Também em concentração de 100 ppm, 26,5\% dos extratos hexânicos e $11,8 \%$ dos extratos etanólicos foram ativos ou parcialmente ativos para desovas, e, $20,6 \%$ dos extratos hexânicos e $5,9 \%$ dos extratos etanólicos foram ativos ou parcial. mente ativos tanto para caramujos adultos como para desovas.

Os autores pretendem fracionar somente os extratos que revelaram-se ativos como moluscicida na concentração de $100 \mathrm{ppm}$. As frações serão submetidas a novos ensaios biológicos em latoratório sobre caramujos adultos, desovas, peixes e camundongos. Somente para as frações tóxicas para caramujos e/ou desovas até $10 \mathrm{ppm}$, para peixes a 100 ppm e não tóxicas para camundongos deverão ser identificadas quimicamente e, posteriormente, testadas no campo em água parada e água corrente. 
MENDES, N. M. et al. Ensaios preliminares em laboratório para verificar a ação moluscicida de algumas espécies da flora brasileira. Rev. Saúde públ., S. Paulo, 18:348 - 54, 1984.

\section{AGRADECIMENTOS}

Ao Prof. José Luiz Pedersoli, do Museu de História Natural da UFMG, pela colabora- ção prestada na classificação botânica de algumas espécies e ao técnico de pesquisas Moacyr Rodrigues da Silva pela coleta das plantas.

MENDES, N. M. et al. [Preliminary laboratory tests of the molluscicide activity of some species of Brazilian flora]. Rev. Saúde Públ., S. Paulo, 18:348-54, 1984.

ABSTRACT: The molluscicide activity of sixty eight extracts from twenty-three Brazilian plants was studied in the laboratory. The solutions, in dechlorinated water, of hexanic and ethylic extracts at 1,10 and $100 \mathrm{ppm}$ concentrations, were tested on adult snails and egg masses of Biomphalaria glabrata, reared in the laboratory. The plants with molluscicide activity on adult snails and/or egg masses at $100 \mathrm{ppm}$ concentration were: Arthemisia verlotorum Lamotte, Caesalpinia peltophoroides Benth, Cassia rugosa G. Don, Eclipta alba Hassk, Euphorbia pulcherrima Willd, Euphorbia splendens Bojer, Joannesia princeps Vell, Leonorus sibiricus L., Macrosiphonia guaranitica Muell, Nerium oleander L., Palicourea nicotianaefolia Cham. and Schlech., Panicum maximum M., Rumex crispus L., Ruta graveolens L. and Stryphno. dendron barbatiman $\mathrm{M}$.

UNITERMS: Biomphalaria glabrata, laboratory conditions. Molluscicide, plant extracts. Schistosomiasis, control.

\section{REFERENCIAS BIBLIOGRÁFICAS}

1. AMORIM, J. P. \& PESSOA, S. B. Experiências de alguns vegetais como moluscicida. Rev. bras. Malar., 14:255-60, 1962.

2. BARBOSA, F. S. \& MELLO, D. A. Ação moluscicida de plantas. Rev. bras. Pesq. méd. biol., 2:364-6, 1969 .

3. KLOOS, H. \& McCULLOUTH, F. Plant molluscicides: a review. Geneva, World Health Organization, 1981. (WHO/SCHISTO/81. 59).

4. PEREIRA, J. P. \& SOUZA, C. P. Ensaios preliminares com Anacardium occidentale como moluscicida. Cienc. Cult., 26:10547,1974 .

5. PEREIRA, J. P.; SOUZA, C. P. \& MENDES, N. M. Propriedades moluscicidas da $E u$ phorbia cotinifolia L. . Rev. bras. Pesq. méd. biol., $11: 345-51,1978$.

6. ROUQUAYROL, M. Z.; SOUSA, M. P. \& SILVA, M. J. M. Atividade moluscicida de plantas do Nordeste brasileiro (III). Rev. bras. Farm., 53:215-20, 1972.

7. ROUQUAYROL, M. Z.; SOUSA, M. P. \& MATOS, F. J. Atividade moluscicida de Pithe- celobium multiflorum. Rev. Soc. bras. Méd. trop., 7:11-9, 1973.

8. ROUQUAYROL, M. Z.; FONTES, M. C.; ALENCAR, J. E.; ABREU MATOS, F. J. \& CRAVEIRO, A. A. Atividade moluscicida de óleos essenciais de plantas do Nordeste brasileiro. Rev. bras. Pesq. méd. biol., $13: 13543,1980$.

9. SILVA, M. J. M.; SOUSA, M.P.\& ROUQUAY ROL, M. Z. Atividade moluscicida de plantas do Nordeste brasileiro (II). Rev. bres. Farm., 52:117-23, 1971.

10. SOUSA, M. P. \& ROUQUAYROL, M. Z. Atividade moluscicida de plantas do Nordeste brasileiro. Rev. bras. Pesq. méd. biol. 7:388-93, 1974.

11. SOUSA, M. P.; ROUQUAYROL, M.Z. \& SILVA, M. J. M, Atividade moluscicida de plantas do Nordeste brasileiro. Rev, bras. Farm., 51:1-9, 1970 .

12. SOUZA, C. P.; AZEVEDO, M. L. L.; LOPES, J. L. C.; SARTI, S. J; SANTOS FILHO, D.; LOPES J. N. C.; VICHNEWSKI, W.; NASI, A. M. T. T. \& LEITÃO FILHO, H. F. Quimioprofilaxia da esquistossomose: atividade moluscicida de produtos naturais 
MENDES, N. M. et al. Ensaios preliminares em laboratório para verificar a açăo moluscicida de algumas espécies da flora brasileira. Rev. Saúde públ., S. Paulo, 18:348 -54, 1984.

- Ensaios com caramujos adultos e deso. vas. An. Acad. bras. Cienc., 1984 [no prelo]

13. WORLD HEALTH ORGANIZATION. Snail control in the prevention of Bilharziasis. Geneva, 1965.

14. WORLD HEALTH ORGANIZATION, Report of the Scientific working Group on Plant
Molluscicide \& Guidelines for evaluation of plant molluscicides. Geneva, 1983. (TDR/SCH-SWE(4)/83.3).

Recebido para publicafāo em 28/11/1983. Reapresentado em 25/06/1984.

A provado para publicaç̃o em 14/07/1984. 